\title{
IN MEMORIAM. FRANCISCO DE ASÍS CHAVERO BLANCO O.F.M. (1945-2001). EL ESTUDIO HECHO ESPÍRITU
}

El día dieciséis de julio de este año, un día después de la festividad de san Buenaventura, este santo franciscano, doctor de la Iglesia, recibió un regalo: una de las personas que aquí y ahora más conocía de su obra, pensamiento y fuentes, iba a ir a visitarlo. Ambos tenían, al fin, la oportunidad de conocerse en persona. Para sus amigos fue un regalo muy caro. Nuestro amigo Francisco de Asís Chavero Blanco nos dejó físicamente, pero sus estudios y su obra perdura y perdurará con nosotros. ${ }^{1}$

Decir que alguien conoce a san Buenaventura no es fácil, pues en este pensador franciscano se conjugan aspectos filosóficos, teológicos, históricos y espirituales que llevan en sí una carga histórica compleja que se desarrolla en un tiempo, a la vez, complejo, apasionante y alejado en el tiempo: el siglo XIII. No en vano, la figura de san Buenaventura fue tildada por algunos como «la más medieval de la filosofía del Medioevo». ${ }^{2}$

Pero Francisco de Asís Chavero se preparó para afrontar este reto y más. Nacido en Rivera del Fresno (provincia de Badajoz) en 1945, profesó en la Orden Franciscana en la Provincia Bética el 6 de agosto de 1967. Se ordenó presbítero el 21 de julio de 1973. Cursó filosofía y teología en el Centro de Estudios Teológicos de Sevilla, en la Facultad de Teología de Granada, en el Pontificio Ateneo Antonianum (Roma) y en el Centro de Investigación de Quarachi de la Orden Franciscana situada en Grottaferrata en Roma. Estos estudios hicieron que quedara corta su titulación de Licenciado en S. Teología, sección Histórico-Dogmática. En el momento en el que le sorprendió la muerte era profesor de Teología Sistemática, Antropología Teológica y Pensamiento Franciscano en el Instituto Franciscano de Murcia.

El perfil de su formación, trabajo y dedicación nos ayuda a comprender por qué a Francisco de A. Chavero no se le escapaba ningún detalle del pensamiento bonaventuriano. No había aspecto de su pensamiento filosófico, teológico, espiritual o franciscano que no conociera. Su obra, sus ediciones, las fuentes, los estudios e interpretaciones sobre el doctor Seráfico eran, no sólo conocidas por él, sino también estudiadas, pensadas e interpretadas. De todo lo que acabo de decir dan fe sus escritos y las extensísimas notas a pie de página que nos regalaba. Hablando de su trabajo siempre mostraba la visión de fondo que impregnaban sus estudios: el de historiador de la teología. Esto no significa que desde esta perspectiva histórica no fuera capaz de percibir y apuntar conceptos nuevos y visiones originales. ${ }^{3}$

Francisco de Asis Chavero Blanco colaboró en numerosas revistas como Verdad y Vida, Carthaginensia, Estudios Franciscanos, Doctor Seraphicus, Themata, Archivun Franciscanum Historicum, etc. Era miembro de la Sociedad Escotista Internacional (Roma), del Centro di Studi Bonaventuriani (Bagnoregio), Asociación Hispánica de Estudios Franciscanos (Barcelona) y compañero de la Sociedad Española de Filosofía Medieval (Zaragoza). ciscano.

Me gustaría en estas páginas repasar aunque sea brevemente el legado intelectual de este pensador fran-

1 Parece que los que aman el estudio y dedican parte de ellos a algún autor en concreto se identifican con ellos sin remedio. Le pasó a más autores, como podemos ver en otro grande: Eusebio Colomer que murió, como nos recuerda Eudaldo Forment, el día de la fiesta del Beato Ramon Llull, a quien había dedicado tantas horas. Cf. Forment, E., «Eusebio Colomer, historiador de la filosofía (1923-1997)», en Revista Española de Filosofía Medieval, 5 (1998), p. 291.

2 Bougerol, J. -G. citado en Piazza, L, Mediazione simbolica in S. Bonaventura. Vicenza, 1978, p. 65.

3 Sobre el carácter filosófico de la obra de Chavero referido a san Buenaventura, publicaremos un artículo que llevará por título «El carácter filosófico de la obra del P. Chavero. Reflexión en torno a la significación filosófica de Imago Deir, que saldrá a la luz próximamente en un número con dos volúmenes dedicado a la memoria del P. Chavero de la revista Carthaginensia, que es órgano de expresión, de investigación y estudios del Instituto Teológico de Murcia (Instituto Franciscano) en el que impartía clases el P. Chavero. 
El día que nos dejó el P. Chavero, a su despacho llegaba recién impreso un escrito de una serie en el que se trataban la vida y obras de algunos autores franciscanos. Uno de ellos, el que se recibió, era el doctor Seráfico, san Buenaventura. A la hora de buscar el autor de la redacción de tal estudio se pensó en alguien que conociera los múltiples aspectos y facetas de su obra, así como el talante propio de la Orden Franciscana. El estudio debía ser sencillo y profundo. Todos sabemos que eso es lo más difícil de realizar. Un estudio erudito es relativamente fácil, porque los lectores ya conocen las problemáticas a tratar. Se trata casi de subrayar matices. Pero explicar a un público en general los entresijos de un período tan complejo, en un autor como san Buenaventura, y hacerlo comprensible, requiere un enorme esfuerzo didáctico y la asunción e interiorización de aquello que se quiere transmitir. El P. Chavero lo consiguió. Y eso sólo es posible conociendo y viviendo los aspectos intelectuales (filosóficos y teológicos), históricos y espirituales de san Buenaveatura y de la Orden Franciscana.

Mi experiencia personal así lo atestigua. Corría el curso 1991-1992 cuando un joven estudiante, entonces de teología, con inquietudes de entrega (faceta que sigo cuidando con mayor o menor acierto) desde el carisma franciscano, realizaba su postulantado en el convento del Santuario de Loreto en Espartinas (Sevilla). Entonces, yo estaba ávido de conocimiento de san Francisco. En el refectorio una animada charla sobre el franciscanismo me puso en guardia ante un fraile dicharachero y guasón, que con gracia y profundidad, discutía sobre la espiritualidad franciscana con una erudición que me sorprendió ya entonces. Me pegué a él y me mostró las fuentes, la bibliografía, los esquemas, la historia y me enseñó la riqueza de la Orden franciscana y del pensamiento medieval. Teníamos la suerte de que en el convento estaba la nutrida biblioteca provincial.

La fortuna, que la vida y Dios me regalaron, de disfrutar de su vasta cultura medieval y franciscana se reflejó en su obra donde aparecen sus inquietudes. Hagamos un repaso a los temas más sobresalientes tratados por el P. Chavero.

\section{ESTUDIOS BONAVENTURIANOS}

El doctor franciscano es quizás el hilo conductor de la obra del P. Chavero. No el único, pero sí el más importante. El estudio bonaventuriano sirvió al P. Chavero a profundizar en los aspectos intelectuales y espirituales de los primeros tiempos de la Orden de Frailes Menores. Los primeros estudios que tengo de mi amigo, aquellos escritos por un joven en la década de los ochenta, tienen al doctor Seráfico como centro de su investigación. Es el caso de su estudio «Teoría bonaventuriana de la redención. Sus presupuestos antropológicos» ${ }^{4}$ El valor de este artículo en la comprensión de su producción ès que muestra otra de las claves de su pensamiento: la antropología teológica. Esta doble preocupación temática culminará en 1993 con la obra recopilatoria Imago Dei. Aproximación a la antropología teológica de san Buenaventura. ${ }^{5}$ Sobre este libro hablaré un poco más tarde.

Los estudios bonaventurianos han sido prolíficos y abundantes. Estos trabajos e investigaciones no sólo se han referido a los distintos aspectos de su pensamiento, sino que han intentado, también fijar su obra, acrecentándose esta labor a través de sus ediciones críticas. El P. Chavero no fue ajeno ante esta ingente labor.

El artículo «El catálogo de las obras de san Buenaventura, estado actual de la cuesti@n», 6 publicado en 1998, aborda la cuestión que suscita la fijación definitiva de los textos bonaventurianos, a través de la relectura desde la crítica textual actual, sobre la edición de los PP. Editores de Quaracchi, cerrando problemas sobre la autenticidad de muchos de ellos y apuntando soluciones o métodos de trabajo sobre los problemas que esta crítica hace aflorar. Este artículo refleja la seriedad de la metodología del trabajo del P. Chavero, quien hace una enumeración exhaustiva de las obras revisadas. Se aprecia, también, el contacto personal del autor con una

4 Chavero Blanco, F. De A., «Teoría bonaventuriana de la redención. Sus presupuestos antropológicos», en Estudios Franciscanos, 86 (1985), pp. 515-576.

5 Id., Imago Dei. Aproximación a la antropología teológica de san Buenaventura, Murcia, Editorial Espigas, 1993.

6 Id., «El catálogo de las obras de san Buenaventura, estado actual de la cuestión», en Carthaginensia, 14 (1998), pp. 43-100. 
institución que supo y sabe aunar el interés inherente de la problemática filosófico-teológica de los autores escolásticos, con el de la importancia de las ciencias históricas. Nos referimos al «Collegio San Buenaventura» de Quaracchi en el que residió durante varias ocasiones.

La elaboración de la lista completa de la obra bonaventuriana efectuada por los PP. Editores de Quaracchi resultaba satisfactoria desde la crítica textual de la época. Sin embargo, a principios del siglo XX aparecen, en torno a las denominadas Quaestiones disputatas, una serie de obras supuestamente bonaventurianas.

Ya hemos visto como el P. Chavero afrontó en 1998 esta cuestión. Pero, más allá aún, nos desenmascara la no-autenticidad bonaventuriana de una de esas cuestiones (La Quaestio de Imagine Recreationis del ms. Assisi, comunale, 186) datándola como anterior a $1245 .{ }^{7}$ En la realización de este estudio se puede apreciar el conocimiento profundo de los conceptos derivados de la teología de la gracia referidas a la antropología teológica medieval.

Del estudio de las fuentes de san Buenaventura surge una riqueza que el P. Chavero supo aprovechar con acierto. Podemos citar, como ejemplo, el artículo titulado: El sermón Iste pauper clamavit y la teología de san Buenaventura». ${ }^{8}$ En el mismo muestra, tomando como hilo conductor el análisis de este sermón falsamente atribuido al doctor Seráfico, la influencia de san Buenaventura en los predicadores y teólogos (en este caso relativos a la teología de la pobreza) de finales del siglo XIII y principios del siglo XIV.

El estudio de los textos nos llevan a la implicación franciscana de un pensador que, no en vano, fue el séptimo General de la Orden de Frailes Menores, llegando a recibir por parte de algunos autores el calificativo de refundador de la Orden. ${ }^{9}$ En un estudio sobre la mirada de san Francisco realizada por parte de san Buenaventura en el Sermo $V$ «Discite me», ${ }_{10}^{10} \mathrm{el}$ P. Chavero descubre la faceta espiritual y franciscana del santo Franciscano, quien nos presenta una imagen teológica del Fundador, propios de su riqueza intelectual. El artículo termina con unas líneas que ya constituyen un reto para otros estudiosos del pensamiento franciscano: «En trabajos sucesivos, escribe el P. Chavero, habrá que continuar el estudio de estos problemas». ${ }^{1 "}$ Este era un tema ya tratado en sus más recientes escritos. ${ }^{12}$

Este dicurso, fuente de la Leyenda maior, apunta a la visión alternativa que propone el santo Franciscano frente a la filosofía llegada y convulsionadora del siglo: la aristotélica. De este modo, el artículo «Vir hierarchicus (Legenda maior, Prologus), ¿Una interpretación de san Francisco en clave dionisiana?», ${ }^{13}$ nos señala la otra fuente neoplatónica del pensamiento bonaventuriano: la del Pseudo-Dionisio. Este artículo nos muestra la labor de síntesis bonaventuriana, a través de la expresión aparecida en el prólogo de la Legenda maior: vir hierarchicus, referida a san Francisco. En esta expresión se puede apreciar la lectura dionisiana que realiza san Buenaventura, interpretada desde su propia teología de la gracia y de su teología espiritual cargada de la tradición franciscana ya existente y el agustinismo medieval..

Por lo visto hasta ahora, el legado que nos lega el P. Chavero referente a los estudios bonaventurianos, los podemos resumir, a riesgo de no ser exhaustivos, en tres aspectos generales:

1) El carácter teológico-espiritual. San Buenaventura es un teólogo que trata de plasmar en sus obras la perspectiva y profundidad teológica. Sus escritos beben de las fuentes de la teología, y su trabajo será, en relación al pensamiento de la Orden Franciscana, en particular de su fundador, más hagiográfico que historiográ-

7 Id., «La Quaestio de Imagine Recreationis del ms. Assisi, comunale, 186. ¿Un escrito bonaventuriano?», en Archivum Franciscanum Historicum, 92 (1999), pp. 3-58.

8 Id.., «El sermón «Iste pauper clamavit» y la teología de san Buenaventura», en ll Santo, 39 (1999), pp. 127-167.

9 Cf. Rogen, H., «Saint Bonaventure, second fondateur de l'Ordre des Frères Mineurs», en Etudes Franciscaines, 17 (1967), p. 78. Esta afirmación se matiza en Desbonnets, Th., De la intuición a la institución, Oñati, Ed. Franciscana Aranzazu, 1991, p. 165.

10 Chavero Blanco, F. de A., «Discite me». El sermo V de Sancto Francisco y la teología de san Buenaventura», en Carthaginensia, 16 (2000), pp. 275-322.

$11 \quad$ Ibid., p. 322.

12 Recién ordenado sacerdote escribe el artículo: Id., «Reflexiones sobre la "Legenda maior sancti Francisci", escrita por san Buenaventura», en Verdad y Vida, 32 (1974), pp. 629-638.

13 Id., «Vir hierarchicus (Legenda maior, Prologus), ¿Una interpretación de san Francisco en clave dionisiana?», en Il Santo, 40 (2000), pp. 7-48. 
fico. Esta lectura franciscana la podemos ver igualmente en el relato de la conversión del fundador que realiza el doctro Seráfico en la Leyenda maior: ${ }^{14}$.

2) El pensamiento teológico tiene como fuente la rica tradición neoplatónica en su doble fuente agustiniana y dionisiana. El baluarte de san Agustín proporciona la estructura y la profundidad necesarias a toda especulación, pues se apoyan en el Logos-Verdad, en Dios. El neoplatonismo en su camino dionisiano ayuda al hombre desde la especulación a la contemplación mística de la fuente de toda sabiduría elevando el espíritu hacia la fuente de toda teología: es un camino de ida y vuelta a Dios, completando y perfeccionando el intento de conciliación ya iniciado por los victorinos, en especial por Hugo de San Víctor.

3) San Buenaventura, en este intento de conservación teológico-espiritual, se mantiene fiel a las tradiciones agustinistas desde la lectura que le proporcionan los que con él comparten su vocación, la Escuela Franciscana de París. Este es el punto de inflexión, la referencia en la cual interpretar su polémica relación con el pensamiento aristotélico.

Estos aspectos claves del pensamiento y la especulación bonaventuriana, los muestra Chavero de modo privilegiado en lo que se refiere a la antropología teológica del doctor Seráfico, conjugando para ello las perspectivas filosófica, teológica, espiritual y franciscana. No en vano, el P. Chavero llevaba años preparando su tesis sobre la antropología teológica (si bien él estaba ya, de sobra, doctorado en conocimientos), cuyo manuscrito me enseñó entusiasmado, la última vez que le visité, ya prácticamente completo. Un ejemplo, de esfuerzo sintético, a la par que denso; respecto a la antropología teológica y sus múltiples facetas, lo desarrolló en su obra más representativa: Imago Dei. Aproximación a la antropología teológica de san Buenaventura. Este libro era el compendio de una serie de artículos aparecidos en distintas publicaciones y que engarza en torno a dos ejes, dos partes. La primera parte bajo el epígrafe Aproximación a la Antropología en la que aparecen los artículos siguientes:

— «Ser y significar. Aproximación al simbolismo bonaventuriano», en Thémata, 5 (1988), pp. 51-71.

— «El hombre y su dimensión de futuro. Para una relectura bonaventuriana», en Chavero Blanco, F. De A. (dir.), en Bonaventuriana. Miscellanea in onore di Jacques Guy Bougerol, 2 vol., Roma, 1988, vol. I, pp. 223-256. En esta obra fue también autor de la «Presentación », pp. 5-7 y I. ${ }^{15}$

- «Per una teología e antropologia dell'immagine di san Bonaventura», en Doctor Seraphicus, 37 (1990), pp. 5-35.

- «Imago creationis, imago recreationis. Para una teología y antropología de la imagen de Dios en san Buenaventura ", en Carthaginensia, 9 (1993), pp. 79-149.

La segunda parte se titula: Implicaciones de la antropología, en la que cual se muestran varios artículos:

— «Sobre el conocimiento de Dios. Posibilidades y límites de la razón», en Actas del Primer Congreso Nacional de Filosofía Medieval, (Zaragoza 12-14 diciembre 1990), Zaragoza, ed. Jorge M. Ayala Martínez, Ibercaja, 1992, pp. 233-247.

— «Teología y antropología», en Estudios Franciscanos, 89 (1988), pp. 229-277.

— «Sobre la filosofía bonaventuriana. Hacia un ensayo de interpretación», en Carthaginensia, 2 (1986), pp. 55-80.

El carácter propio del libro muestra el carácter antropológico en las facetas que habíamos comentado, a saber: filosófico, teológico, espiritual y franciscano. Por el carácter de estas líneas tan sólo me permito reproducir algunas frases que el P. J. -G. Bougerol manifestaba en la presentación de esta obra: «Alors on peut parler de la Théologie et de l'Anthropologie fondées sur l'exemplarisme» (en relación al fundamento filosófico); «Homme de désir, homme du futur tendu vers la réalisation de la béatitude, il entre à part entière dans le grand projet d'amour du Verbe Incarné qui devient l'homme créé par lui, pour sauver ce qui en lui est perdu. Il faut méditer les pages substantielles où Francisco Chavero décrit le "Sacramentun futuri") (en relación a la lectura teológica y la doctrina de la redención y la gracia); «Nous pourrions dire aujourd'hui que l'homme est un

14 Id., «Immutatio dexterae altissimi. Lectura teológica de la conversión de san Francisco (Legenda Maior Cap. I)», en Antonianum, 75 (2000), pp. 681-719.

15 Este artículo conocerá una relectura posterior en un artículo aparecido cinco años después: Id., «Antropología y escatología. Ensayo de una re-lectura bonaventuriana», en Naturaleza y Gracia, 45 (1998), pp. 255-332. 
pélerin en chemin vers Dieu, ce qui équivaut à souligner son caractère eschatologique» (subrayando el carácter espiritual); por último un sentido homenaje al franciscano que piensa y que era (y es) el P. Chavero, y que el P. Bougerol expresa con estas sentidas palabras «Dans ce volume, Francisco Chavero a mis en pleine lumière l'un des problèmes fondamentaux de la pensée bonaventuirenne. Il l'a fait avec une clarté et une précision que j'ai plaisir à saluer fraternellement, répondant ainsi à celui qui avait pris en charge et présenté l'hommage des Bonaventuriana». ${ }^{16}$

Esta última cita me lleva a culminar este breve repaso sobre sus estudios bonaventurianos con el homenaje que brindó en forma de Miscellanea al P. Bougerol. Bonaventuriana. Miscellanea in onore di Jacques Guy Bougerol. Es una obra que dirigió con mucho cariño el P. Chavero al gran «Maestro» de los estudios bonaventurianos y, diría yo que los que hemos tenido la oportunidad de hablar con él, mejor persona aún, el $P$. Bougerol. Esta obra, creo, supone su madurez intelectual. Significa un reto importante para él, que le llenaba de orgullo. No sólo porque es una obra que recoge en tres partes 34 artículos bonaventurianos de autores incontestables — cabe citar (siendo injusto porque todos ellos son sobresalientes), además del propio Chavero, a Robert Javelet, Paul Vignaux, Isidoro Manzano, José Antonio Merino, etc.-, sino porque fue una aportación, bajo su responsabilidad, de un encargo del entonces secretario para el Oficio de la Formación y Estudios de la Orden, el P. Hermann Schlük. Y este fue su principal apostolado: el estudio y la transmisión de los valores de la Orden de Frailes Menores. Pero es que, además, esta obra señala esos aspectos a los que ya nos hemos referido en todas estas líneas: las fuentes, el pensamiento filosófico, teológico y espiritual, el carácter franciscano y la influencia del doctor Seráfico, que era para él el Maestro.

No me resisto a terminar sin citar un artículo relativamente reciente, del año 2000, que bajo el título «Initium Salutis. Aproximación a la teoría bonaventuriana de la creación» ${ }^{17}$ afronta la significación creatural desde los aspectos simbólicos e históricos, y su significación metafísica desde la fuente Trinitaria, que resume también su visión del mundo que rodea la antropología; el contexto en el que está inmerso: el mundo creado.

\section{OTROS ESTUDIOS}

El P. Chavero centró su obra en san Buenaventura, pero no renunció a realizar otros estudios, que si bien siempre giraron en torno a la orden franciscana, podríamos dividir en dos senderos: 1) El contexto franciscano del pensamiento bonaventuriano; 2) El estudio del arte y la historia franciscana de la Provincia a la que pertenecía: la Provincia Bética.

De entre los primeros, podríamos citar en torno a las fuentes y los manuscritos, el artículo titulado «La Quaestio de doctrina theologiae del manuscrito Vat. Lat. 782. Introducción y edición». ${ }^{18}$ En este estudio, referido al entorno franciscano parisino anterior a 1245, el P. Chavero ensaya un intento de clarificación de este manuscrito que intepreta como misceláneo (dos manos parecen al menos haberlo compuesto), heredero de la concepción teológica de Hugo de S. Víctor. Fuera de esa conclusión, se podrían tachar de «hipótesis de trabajo» las conclusiones dadas al respecto por autores como Bougerol, Pelster o Doucet que lo atribuyen a Juan de la Rochella o Alejandro de Hales. Chavero señala, a su vez, los paralelismos del texto con el «Tractatus Introductorius» de la Summa Halensis. En definitiva, este artículo supone un paso más en la comprensión de las fuentes franciscanas en general, así como su teología y pensamiento.

Precisamente, sobre Juan de la Rochella había escrito, cuatro años antes, un estudio bajo el título «Finalidad del estudio de la teología. En torno a un texto de Juan de la Rochella», ${ }^{19}$ en los que también inves-

16 Bougerol, J. -G., «Presentación», en Chavero Blanco, F. De A., o.c., pp. IX-XII.

17 Chavero Blanco, F. De A., «Initium Salutis. Aproximación a la teoría bonaventuriana de la creación», Estudios Franciscanos, 101 (2000), pp. 1-72.

18 Id., «La Quaestio de doctrina theologiae del manuscrito Vat. Lat. 782. Introducción y edición», en Carthaginensia, 15 (1999), pp. 31-72.

19 Id., «Finalidad del estudio de la teología. En torno a un texto de Juan de la Rochella», en Carthaginensia, 11 (1995), pp. 35-80. 
tiga su relación con Alejandro de Hales, quien eclipsó su figura. Chavero nos ilumina lo que ocultó, por motivos ajenos a él, el autor de la Summa Halensis.

Y su interés por las fuentes, recordemos, estaba al servicio de la antropología teológica, que es lo que intenta recuperar en un estudio franciscano sobre Odón Rigaldo. ${ }^{20}$

Pero, además de artículos relacionados con el tema teológico bonaventuriano y franciscano, también se interesó por la historia y el arte franciscanos en la Provincia Bética. A este respecto podemos citar un artículo que sirve de gozne entre ambos intereses intelectuales. Publicado en las actas del Congreso Escotista Internacional celebrado en marzo de 1993, Chavero escribe un estudio referido a la influencia del escotismo en la Provincia Bética Franciscana cuando era profesor del Instituto Teológico de Sevilla. El estudio, que lleva por título «Sobre el escotismo en la Provincia Bética. El ocaso de una tradición» ${ }^{21}$ nos muestra su faceta más historiadora en su análisis de los datos, por dificultosos que fueran. Como él mismo manifiesta: «Si la historia de los estudios en la Provincia Bética Franciscana es una labor casi irrealizable por la ausencia de una documentación, en este erial he podido encontrar un dato ciertamente exiguo, pero a mi juicio significativo: una disposición capitular que prescribe un cambio de estudios». ${ }^{22}$

Este ámbito de estudio histórico-franciscano que caracterizaba, al P. Chavero, tuvo como marco excepcional los Cursos de Verano (Universidad de Verano). La Universidad de Córdoba, junto con la Asociación Hispánica de Estudios Franciscanos, promueven cursos en la localidad de Priego de Córdoba en torno al franciscanismo en el ámbito andaluz, denominados: El Franciscanismo en Andalucía. De hecho, Chavero estaba preparando, con la ilusión que le caracterizaba, la ponencia «La plata en el convento de Loreto» - convento en el que residía y en el que convivimos juntos un año-. De estas jornadas estivales tengo en mi mesa de estudio tres de sus intervenciones que me gustaría, al menos, citar, y que dan fe de lo que acabo de decir. En el II Curso de Verano, que giraba en torno a «San Francisco en la Historia y en el arte andaluz», el P. Chavero nos regaló una preciosa lectura iconográfica hecha desde el Barroco, que aúna conocimiento artístico y teológico de gran altura: «La Iglesia conventual de Santa Clara de Sevilla. Propuesta de Lectura iconológica».23 Lectura iconológica que repitió el curso siguiente sobre uno de los conventos franciscanos que le era más querido: «La Iglesia del Colegio de San Buenaventura de Sevilla. Lectura iconológica». ${ }^{24}$ En ella muestra sus conocimientos de la obra de Francisco Herrera, el Viejo, realizando una lectura de sus frescos que giran en torno a la armonía fe-razón; respondiendo así, desde una interpretación concreta, a lo que el lema del curso reclamaba ese año: «San Francisco en la cultura y en la historia del arte andaluz». El Cuarto Curso de Verano, titulado: «San Francisco en la cultura andaluza e hispanoamericana», fue una oportunidad para conocer la figura «grande» del fraile Fray Bernardino de Laredo ${ }^{25}$-franciscano, médico y escritor-, por medio del estudio de su obra «Subida al Monte Carmelo». Una obra ascético-mística de gran amplitud antropológica en la tradición del «socratismo cristiano», según una expresión de E. Gilson. ${ }^{26}$

Termino con un estudio que vincula su vocación franciscana (teológica y èspiritual) con la tierra que le vio nacer: Extremadura. Con motivo de la celebración del Quinto Centenario del nacimiento de San Pedro de

20 Id., «Homo, imago Dei. En torno a un texto de Odón Rigaldi», en P. Llorente et.al, El pensament antropologic medieval, Vic, 1996, pp. 228-238.

21 Id., «Sobre el escotismo en la provincia Bética. El ocaso de una tradición», en Leonardo Sileo (dir.) Via Scoti. Methodologica ad mentem Joannis Duns Scoti. Atti del Congresso Scotistico Internazionale. Roma 9-11 marzo 1993 (2vol.), Vol. 2, Roma, Edizioni Antonianum, 1995, pp. 1171-1197.

22 Ibíd.., p. 1171.

23 Id., «La Iglesia conventual de Santa Clara de Sevilla. Propuesta de Lectura iconológica», en Peláez del Rosal, M. (dir. y ed.), El franciscanisno en Andalucía: San Francisco en la historia y en el arte andaluz, Córdoba, Peláez del Rosal (ed. especial), 1998, pp. 51-92.

24 Id., "La Iglesia del Colegio de San Buenaventura de Sevilla. Lectura iconológica», en Peláez del Rosal, M. (dir. y ed.), El franciscanismo en Andalucía: San Francisco en la cultura y en la historia del arte andaluz, Córdoba, Peláez del Rosal (ed. especial), 1999, pp. 55-84.

25 Id., «Fray Bernardino de Laredo y su Subida al Monte Carmelo. Una visión cristiana del hombre», en Peláez del Rosal, M. (dir. y ed.), El franciscanismo en Andalucia: San Francisco en la cultura andaluza e hispanoamericana, Córdoba, Peláez del Rosal (ed. especial), 2000, pp. 55-84.

26 Cf. Gilson, E., El espiritu de la filosofia medieval, Madrid, Ed. Gredos, pp. 213-231. 
Alcántara, el P. Chavero dictó la ponencia «San Pedro de Alcántara: Su doctrina espiritual en el ámbito de la teología franciscana», ${ }^{27}$ un sentido estudio en el que relucen las virtudes que hemos intentado reseñar en estas páginas, y en el cual recalca la pertenencia de este gran «Hombre Universal», que fue el santo alcantarino, a la fuente espiritual que lo conforma: San Francisco de Asís y la tradición parisina del siglo XIII, en especial la obra espiritual bonaventuriana.

La revista Carthaginensia dedicará un número especial a la memoria del padre Chavero, en el cual se incluirá la Quaestio de Beatitudine, introducción y edición.

\section{FRANCISCANO}

Debemos a san Francisco muchas excelencias de carácter espiritual, de espíritu constructivo, de lucha por la paz y el equilibrio con el mundo y sus creaturas. Pero, sobre todo, le debemos el hecho de impulsar, respondiendo generosamente a la llamada del Espíritu, un movimiento que nos ha dejado un reflexión profunda. Un movimiento espiritual que ha enriquecido el propio espíritu original del Poverello, y que ha hecho más profundo el misterio humano y su relación trascendente. Una Orden, la Franciscana, que nos dejó, desde el siglo XIII en que vio la luz, grandes hombres, grandes gestas espirituales e intelectuales. No sólo lo que implicó la Escuela Franciscana de París (Alejandro de Hales, San Buenaventura o Duns Scoto, por mencionar algunos), o las grandes almas espirituales de la Edad de Oro (Fray Luis de Granada, San Pedro de Alcántara), sino también estudiosos que en aras al amor a la Orden y la llamada a la búsqueda de la comprensión a la Verdad a la que se han consagrado, nos han donado hermosas páginas, reflexiones y un amor al trabajo y al estudio del pensamiento medieval. Nosotros hemos tenido el placer de compartir su sabiduría, no sólo en sus escritos, sino también en su conversación. En dos años se han ido el P. Rivera de Ventosa y, ahora, el P. Chavero. Dos franciscanos amantes de san Francisco y del estudio medieval.

Manuel Lázaro Pulido
Instituto Superior de Ciencias Religiosas «Santa María de Guadalupe»
«Casa de la Iglesia»
c/ Ramón Albarrán, 36
$06002-$ Badajoz

27 Chavero Blanco, F. de A., «San Pedro de Alcántara: Su doctrina espiritual en el ámbito de la teología franciscana», en García, S., San Pedro de Alcántara, Hombre Universal. Congreso de Guadalupe, 1997, Madrid, Ed. de Guadalupe, 1998, pp. 381-437. 